\title{
EVALUACIÓN DE ÍNDICES FISIOLÓGICOS EN TERNE- ROS LACTANTES DURANTE DOS INVIERNOS EN LA CUENCA LECHERA SANTAFESINA
}

\author{
LeVA, P. E. ${ }^{1} ;$ Garcia, M. S. ${ }^{\text {; }}$ SOSA, J. ${ }^{1}$; \\ Toffoli, G. ${ }^{1}$; Fernandez, G. ${ }^{2}$ \& Valtorta, S. E. ${ }^{3}$
}

\begin{abstract}
RESUMEN
Con la finalidad de identificar índices fisiológicos que puedan ser utilizados como indicadores de bienestar para terneros Holando en su etapa lechal, se llevo a cabo una experiencia durante dos inviernos. Se utilizaron 12 terneros cada invierno, en dos sistemas de crianza diferentes: a) en estaca y b) estaca modificado y provisto de capas de protección. Los terneros recibían 4 litros de leche por día, balanceado iniciador y agua ad libitum. Se pesaron los animales dos veces: al nacer y a los 50 días. Cada 15 días y 4 veces se realizaron las mediciones de ritmo cardíaco y ritmo respiratorio. Se utilizó el cosinor para detectar ritmos circadianos. Se detectaron diferencias significativas para los dos índices y para ganancia de peso en el primer invierno. Se concluye que el sistema modificado presenta beneficios cuando el invierno es anormalmente frio. El comportamiento de los índices fisiológicos fueron muy diferente en ambos años.
\end{abstract}

Palabras clave: bienestar animal, ritmos circadianos, sistemas de crianza.

\section{SUMMARY}

Evaluation of physiological indexes in suckling calves in the Santa Fe milking area, during winter.

Twenty four suckling Holsetin calves were subjected to trials during two winters, in order to identify physiological indexes that could be utilized as well-being indicators. Twelve animals were included each winter, and were assigned to two rearing systems: a) stake system and b) modified stake + protective capes. All calves received milk (4 L/day) and starter and water ad libitum. Animals wee weighed at birth and at 50 days. Heart and respiration rates were measured once every at 15 days at four different times, to analyze circadian rhythms by cosinor. Significant Differences were detected for both rates and for body weight gain during the first winter. It was concluded that the modified system is advantageous when winters are colder. The physiological parameters responded quite differently during both trials.

Key words: animal wellbeing; circadian rhythms, rearing systems.

1.- Facultad de Ciencias Agrarias, Universidad Nacional del Litoral. Kreder 2805. Esperanza, provincia de Santa Fe.Email: pleva@fca.unl.edu.ar

2.- Facultad de Ciencias Veterinarias, Universidad Nacional del Litoral. Kreder 2805. Esperanza, provincia de Santa Fe.

3.- Consejo Nacional de Investigaciones Científicas y Técnicas (CONICET), con lugar de trabajo en el Instituto de Patobiología, INTA Castelar, Buenos Aires, Argentina. / Facultad de Ciencias Agrarias, Universidad Nacional del Litoral.

Manuscrito recibido el 7 de octubre de 2009 y aceptado para su publicación el 12 de noviembre de 2009 . 